\title{
BOOP: an important cause of migratory pulmonary infiltrates?
}

\author{
T.E. King Jr*
}

Bronchiolitis obliterans with organising pneumonia (BOOP) is a common, nonspecific pulmonary reparative reaction. It is characterized histopathologically by an excessive proliferation of granulation tissue within small airways and alveolar ducts, and is associated with chronic inflammation in the surrounding alveoli. These histopathological findings are identified in many pulmonary processes, including: organizing infections (viral, mycoplasmal, bacterial and fungal); organising diffuse alveolar damage; hypersensitivity pneumonitis; organizing aspiration pneumonia; drug reactions; connective tissue diseases; toxic fume exposures; eosinophilic pneumonia; eosinophilic granuloma; Wegener's granulomatosis; and around other lesions, including abscesses and tumours [1]. The best known syndrome associated with this lesion is cryptogenic organizing pneumonia (COP) or idiopathic BOOP.

Although COP is a diagnosis of exclusion, it is a specific clinicopathological syndrome characterized by a "pneumonia-like" illness without an associated disease [2-10]. There is no gender predilection, and the age at presentation is most often 45-70 yrs. Disease onset is usually within 4-6 weeks of presentation, and is often dramatic, with the development of a flu-like illness, characterised by cough, fever, malaise, fatigue and weight loss. Inspiratory crackles are frequently present on chest examination. Routine laboratory studies are nonspecific $[3,10,11]$. Pulmonary function is usually impaired, with restrictive defect being most common. The transfer capacity of the lungs for carbon monoxide, TLCO, is reduced ( $<80 \%$ of predicted) in three quarters of patients. Hypoxaemia at rest or with exercise is very common.

The roentgenographic manifestations associated with any illness where BOOP is a prominent histopathological lesion are often distinctive, with bilateral, diffuse alveolar opacities in the presence of normal lung volume being characteristic $[4,6,8]$. A peripheral distribution of the alveolar opacities, similar to that thought to be "virtually pathognomonic" for chronic eosinophilic pneumonia, is occasionally seen $[3,8,9]$. Irregular linear or nodular interstitial infiltrates are on rare occasions present as the only radiographic manifestation. Honeycombing is unusual, and is seen only as a late manifestation in the few patients with progressive disease. Other radiographic abnormalities, such as pleural effusion, pleural thickening, hyperinflation and cavities are rare. Computed

*: National Jewish Center for Immunology and Respiratory Medicine, 1400 Jackson Street, Denver, CO 80206, USA. tomography (CT) scans of the lung reveal patchy airspace consolidation, small nodular opacities, and bronchial wall thickening and dilation, most frequently in the periphery of the lung and often in the lower lung zone $[8,12,13]$. Frequently, the CT findings are far more extensive than is expected by review of the plain chest radiograph.

Two papers in this issue of the Journal $[14,15]$ present compelling evidence in support of a role for irradiation injury as a cause of a clinicopathological syndrome similar to idiopathic BOOP. Importantly, these papers present additional evidence that localized lung irradiation can cause bilateral lung disease. Finally, the illness in these patients was characterized by migratory infiltrates; therefore, suggesting that BOOP should be added to the list of causes of migratory infiltrates.

Radiation therapy that includes the lung invariably induces lung damage, with the lesions confined almost exclusively to the radiation port. Often the injury is clinically silent and reversible. The extent and severity of the injury are related to the volume of lung irradiated (the more lung irradiated the greater the likelihood of injury), the total amount of radiation delivered, the rate of delivery (shorter time between treatment is associated with more injury), concomitant chemotherapy, and the concurrent use of corticosteroids [16]. It is unclear what significance can be placed on the fact that the three cases reported in these papers had radiotherapy for carcinoma of the breast $[14,15]$. Clinical radiation pneumonitis is uncommon in this setting, less than $5 \%$ of patients, and is rarely fatal $[17,18]$.

It is rare for "clinically significant" radiation-induced lung injury to occur outside the irradiation port. However, several descriptions of bilateral pulmonary reaction that have following unilateral irradiation have recently been reported (reviewed in $[19,20]$ ). Acute "radiation pneumonitis" occurs 4-12 weeks after treatment is completed. The most common signs and symptoms include dyspnoea, cough, low-grade fever and chest discomfort. Acute radiation pneumonitis is manifested roentgenographically by a subtle, faint indistinctness around vessels, or faint hazy opacities; or may be more marked, with a patchy or relatively homogenous nonsegmental airspace opacities (often with air bronchograms) restricted to the radiation port [20]. CT lung scan, especially high resolution CT (HRCT), is more sensitive than chest radiography in showing these abnormalities [20].

Histopathologically, early radiation injury has the appearance of diffuse alveolar damage (DAD) manifested by 
an acute exudative phase, an organising or proliferative repair phase, and a chronic fibrotic phase [1,20]. During the repair phase, a pattern of organizing pneumonia (i.e. intra-alveolar organization) is prominent and is indistinguishable from that seen in BOOP [1]. Consequently, identification of these lesions depends on the stage when diagnosis is established, i.e. during proliferative repair phase when organizing pneumonia or a "BOOP"-like lesion is commonly present [21]. Acute radiation pneumonitis usually resolves spontaneously or in response to corticosteroid therapy. Chronic radiation fibrosis may follow acute radiation pneumonitis, especially when the acute changes are severe (usually 9-12 months after completion of the treatment). Histopathologically, chronic radiation fibrosis resembles usual interstitial pneumonia with interstitial fibrosis and foci of honeycombing. Therefore, the distinctiveness of the present reports [14, 15] relates primarily to the occurrence outside the radiation port and the recurrent, migratory nature of the lesions.

"Recurrent" pulmonary opacities, i.e. clearing followed by a return of pulmonary opacities in the same location, are recognized in certain diseases: for example, chronic eosinophilic pneumonia; partially treated bacterial pneumonias; situations where endobronchial obstruction impairs airway clearance; and BOOP. Often the recurrence is secondary to cessation of corticosteroid therapy. However, recurrent pulmonary infiltrates are not common following radiation injury. In radiation injury there is usually progression of the lung injury leading to radiation fibrosis.

There are few disease processes where fleeting or migratory pulmonary infiltrates are common. Most frequently, this is seen in syndromes associated with peripheral and/or pulmonary eosinophilia. The best example is simple pulmonary eosinophilia or Loffler's syndrome. This is usually a mild illness associated with migratory pulmonary opacities and a tendency towards spontaneous improvement in 4-6 weeks. Other processes associated with migratory alveolar opacities include: hypersensitivity to drugs; parasitic infection; fungus-induced, especially allergic bronchopulmonary aspergillosis; and Churg-Strauss vasculitis. Migratory pulmonary infiltrates have been noted in several case series of idiopathic BOOP [3, 4, 10, 11, 22-25]. IzUMI et al. [24] reported that $50 \%$ of Japanese patients with idiopathic BOOP had migration of the radiographic shadows. Since the chest radiograph may fail to reveal the full extent of the pulmonary parenchymal involvement in BOOP [25], in some instances the appearance of "new" infiltrates may not be "recurrent" disease but may simply reflect progressive disease not recognised on the plain chest radiograph. Thus, the apparent migratory or fleeting nature of the lesions may represent inadequate treatment of the disease.

In summary, the two reports in this issue of the Journal $[14,15]$ provide further evidence for a role for irradiation injury as a cause of clinicopathological syndrome identical to idiopathic BOOP, i.e. radiation-induced BOOP. In addition, the clear demonstration of migratory pulmonary infiltrates in this setting alerts us to the fact that BOOP should be considered in the differential diagnosis of patients with this clinical picture. This is impor- tant because proper diagnosis will lead to appropriate treatment that will probably result in a cure or control of the disease process in most patients. Importantly, corticosteroid therapy results in complete clinical, radiological and physiological recovery in two thirds of patients with BOOP. In general, clinical improvement is rapid, i.e. within several days or a few weeks. As demonstrated in the cases reported, relapses can occur when the corticosteroids are withdrawn, usually within 1-3 months after therapy is stopped. Most will improve when retreated with corticosteroids.

\section{References}

1. Colby TV, Lombard C, Yousem SA, Kitaichi M. Atlas of pulmonary surgical pathology. Philadelphia, W.B. Saunders, Co., 1991; p. 380.

2. Grinblat J, Mechlis S, Lewitus Z. Organizing pneumonia-like process: an unusual observation in steroid responsive cases with feature of chronic interstitial pneumonia. Chest 1981; 80: 259-263.

3. Davison AG, Heard BE, McAllister WAC, Turner-Warwick MEH. Cryptogenic organizing pneumonitis. $Q \mathrm{~J} \mathrm{Med}$ 1983; 52: 382-394.

4. Epler GR, Colby TV, McLoud TC, Carrington CB, Gaensler EA. Bronchiolitis obliterans organizing pneumonia. $N$ Engl J Med 1985; 312: 152-158.

5. Katzenstein ALA, Myers JL, Prophet DW, Corley LSI, Shin MS. Bronchiolitis obliterans and usual interstitial pneumonia. Am J Surg Pathol 1986; 10: 373-381.

6. Chandler PW, Shin MS, Friedman SE, Myers JL, Katzenstein ALA. Radiographic manifestations of bronchiolitis obliterans with organizing pneumonia versus usual interstitial pneumonia. Am J Roentgenol 1986; 147: 899-906.

7. Guerry-Force ML, Mueller NL, Wright JL, et al. A comparison of bronchiolitis obliterans with organizing pneumonia, usual interstitial pneumonia, and small airways disease. Am Rev Respir Dis 1987; 135: 705-712.

8. Muller NL, Guerry-Force ML, Staples CA, et al. Differential diagnosis of bronchiolitis obliterans with organizing pneumonia: clinical, functional and radiologic findings. Radiology 1987; 162: 151-156.

9. Bartter T, Irwin RS, Nash G, Balikian JP, Hollingsworth $\mathrm{HH}$. Idiopathic bronchiolitis obliterans organizing pneumonia with peripheral infiltrates on chest roentgenogram. Arch Intern Med 1989; 149: 273-279.

10. Cordier JF, Loire R, Brune J. Idiopathic bronchiolitis obliterans organizing pneumonia: definition of characteristic clinical profiles in a series of 16 patients. Chest 1989; 96: 999-1004.

11. Yamamoto M, Ina Y, Kitaichi M. Bronchiolitis obliterans organizing pneumonia (BOOP): profile in Japan. In: Harasawa M, Fukuchi Y, Morinari H, eds. Interstitial pneumonia of unknown etiology. Tokyo, University of Tokyo press, 1989, pp. 61-70.

12. Nishimura K, Itoh $\mathrm{H}$. Is $\mathrm{CT}$ useful in differentiating between BOOP and idiopathic UIP? In: Harasawa M, Fukuchi Y, Morinari H, eds. Interstitial pneumonia of unknown etiology. Tokyo, University of Tokyo press, 1989, pp. 317-324.

13. Muller N, Staples C, Miller R. Bronchiolitis obliterans organizing pneumonia: CT features in 14 patients. Am J Roentgenol 1990; 154: 983-987. 
14. Crestani B, Kambouchner M, Soler P, et al. Migratory bronchiolitis obliterans organizing pneumonia after unilateral radiation therapy for breast carcinoma. Eur Respir $J$ 1995; 8: 318-321.

15. Bayle JY, Nesme P, Bejui-Thivolet F, Loires R, Guerin JC, Cordier JF. Migratory cryptogenic organizing pneumonitis "primed" by radiation therapy. Eur Respir $J$ 1995; 8: 322-326.

16. Rosenow EC III, Martin WJ II. Drug-induced interstitial lung disease. In: Schwarz MI, King TE Jr, eds. Interstitial lung disease. 2nd edn. St. Louis, Mosby Year Book, 1993, pp. 255-270.

17. Cherniack RM, Abrams J, Kalica AR. Pulmonary disease associated with breast cancer therapy. Am J Respir Crit Care Med 1994; 150: 1169-1173.

18. Kimsey FC, Mendenhail NP, Ewald LM, Coons TS, Layon AJ. Is radiation treatment volume a predictor for acute or late effect on pulmonary function? Cancer 1994; 73: 2549-2555.

19. Gibson PG, Bryant DH, Morgan GW, et al. Radiation- induced lung injury: a hypersensitivity pneumonitis? Ann Intern Med 1988; 109: 288-291.

20. Davis SD, Yankelevitz DF, Henschke CI. Radiation effects on the lung: clinical features, pathology and imaging findings. Am J Roentgenol 1992; 159: 1157-1164.

21. Kaufman J, Komorowski R. Bronchiolitis obliterans. A new clinical-pathologic complication of irradiation pneumonitis. Chest 1990; 97: 1243-1244.

22. Miyagawa Y, Nagata N, Shigematsu N. Clinicopathological study of migratory lung infiltrates. Thorax 1991; 48: 233-238.

23. Epstein DM, Bennett MR. Bronchiolitis obliterans organizing pneumonia with migratory pulmonary infiltrates. Am J Roentgenol 1992; 158: 515-517.

24. Izumi T, Kitaichi M, Nishimura K, Nagal S. Bronchiolitis obliterans organizing pneumonia: clinical features and differential diagnosis. Chest 1992; 102: 715S-719S.

25. Nishimura K, Itoh H. High-resolution computed tomographic features of bronchiolitis obliterans organizing pneumonia. Chest 1992; 102: 26S-31S. 